\title{
SENSITIVITY ANALYSIS FOR PARAMETERS OF PRESTRESSED CONCRETE BRIDGE USING NEURAL NETWORK ENSEMBLE
}

\author{
LX. Pan ${ }^{*}$, D. Lehký ${ }^{* *}$, D. Novák ${ }^{* * *}$, M. Cao ${ }^{\dagger}$
}

\begin{abstract}
Structural reliability assessment is imperative to keep structural safety, durability and serviceability. One vital factor of such assessment is determination of dominant parameters of structure, called sensitivity analysis. There are many methods for determining dominant parameters, among them artificial neural networks are superior. Existing methods are generally based on a single neural network, but inadequate as a basis for parameter sensitivity analysis because of the instability of a single neural network. To address this deficiency, the paper describes a neural network ensemble-based parameter sensitivity analysis. The proposed method is applied to prestressed concrete bridge. Three dominant parameters were identified for limit state of decompression and six dominant parameters for limit state of crack initiation. The proposed method provides a common paradigm for analyzing the sensitivity of influential parameters, providing effective information to set up models and even to simplify reliability assessment.
\end{abstract}

Keywords: reliability, sensitivity analysis, neural network-ensemble, prestressed concrete bridge

\section{Introduction}

Sensitivity analysis is a crucial step in computational modeling and assessment. Several sensitivity analysis methods have been developed, giving rise to a vast and growing body of literature, e.g. Kleijnen (2010), Borgonovo and Plischke (2016). Structural reliability assessment is imperative to keep structural safety, durability and serviceability. In the process of structural reliability assessment, a vital step is obtaining the dominant parameters, e.g. influential input parameters of stochastic model, key parameters effecting on loading-bearing capacity. There are many methods for determining dominant parameters, among them artificial neural networks are superior. Existing methods are generally based on a single neural network, but inadequate as a basis for parameter sensitivity analysis due to the uncertainty of neural network modelling (Benaouda et al., 1999, Maier et al., 2000), mainly resulting from (1) the lack of a reliable method for determining the structure of the hidden layer, (2) the dissimilar properties of various learning algorithms used to train the neural networks, (3) the lack of a theoretical guide for choosing optimal performance criteria to direct the training of neural networks and (4) random initialization of connection weights inducing uniqueness of the model. To address this deficiency, a neural network ensemble-based parameter sensitivity analysis paradigm has been developed (Cao et al., 2017). This paradigm uses a group of preselected superior neural networks to independently carry out neural network modelling, subsequently performing parameter sensitivity analysis and finally collectively making decisions as to the sensitivity of parameters by summarizing the sensitivity analysis results of individual neural networks. The efficiency of the proposed paradigm is illustrated on a prestressed concrete bridge example (Lehký et al., 2017).

\footnotetext{
Lixia Pan, PhD Candidate: Institute of Mechanics and Materials, Hohai University, No.8 Focheng West Road; 211100, Nanjing; CN, plxxak@163.com

** David Lehký, associate professor, Institute of Structural Mechanics, Faculty of Civil Engineering, Brno University of Technology, Veveří 95, 66237 Brno; CZ, lehky.d@fce.vutbr.cz

*** Drahomír Novák, professor, Institute of Structural Mechanics, Faculty of Civil Engineering, Brno University of Technology, Veveř́ 95, 66237 Brno; CZ, novak.d@fce.vutbr.cz

$\dagger \quad$ Maosen Cao, professor: Institute of Mechanics and Materials, Hohai University, No.8 Focheng West Road; 211100, Nanjing; CN, cmszhy@163.com
} 


\section{Sensitivity method}

The sensitivity approach is based on an artificial neural network (ANN). ANNs are powerful, flexible, versatile techniques which are often employed in the solution of various types of engineering problems including prediction, classification and approximation. Apart from these prevailing applications, the use of ANNs to perform parameter sensitivity analysis for engineering systems is still uncommon, although the huge potential of ANNs has become evident in this area of research.

Existing studies, mostly describe the application of a single artificial neural network. It is difficult, however, for a single neural network to arrive at an accurate result concerning parameter sensitivity. This is due to the uncertainty of neural network modeling. To address this deficiency, neural network ensemble-based (NNE) sensitivity analysis is used (Cao et al., 2017). In this approach, a group of preselected neural networks is trained to independently carry out neural network modeling and perform parametric sensitivity analysis. NNE-based parameter sensitivity analysis is designed to overcome the deficiencies of existing ANN-based parameter sensitivity analyses. The procedure involves four basic steps:

1. A sophisticated type of neural network is empirically chosen as the seed;

2. the seed produces a family of $\mathrm{k}$ candidate neural networks with dissimilar network structures;

3. with the removal of inferior neural networks, $n(n \leqslant k)$ superior neural networks with better performance are selected to form an NNE model;

4. a selected sensitivity analysis algorithm is implemented on the NNE model to perform parameter sensitivity analysis, leading to the ranking of sensitivity for all the parameters pertaining to the engineering system of interest.

Fig. 1 shows a schematic of NNE-based parameter sensitivity analysis. The components are clearly identified, i.e. the seed, candidates, NNE model and ranking.

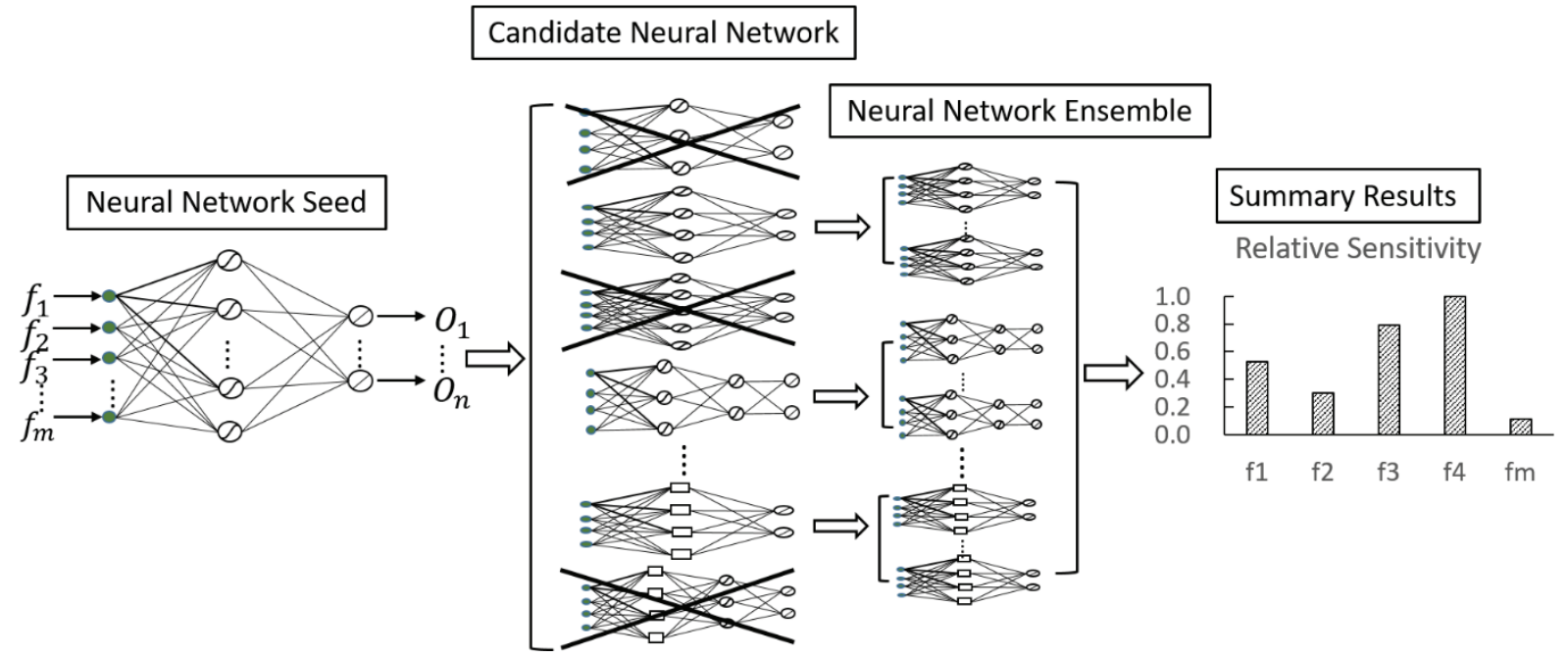

Fig. 1: A schematic view of NNE-based parameter sensitivity analysis

Apart from network structures, sensitivity analysis algorithms also somewhat influence the results of NNE-based parameter sensitivity analysis. Various sensitivity analysis algorithms can be found in the literature, e.g. the partial derivatives algorithm, the input perturbation algorithm and the connection weights algorithm. Among these algorithms, the most widely used is the input perturbation algorithm, which provokes a change or perturbation in the input, consists of comparing the mean square error made by the network from the original patterns with the error made when restricting the input of interest to fixed value for all patterns. Thus, the greater the increase in the mean square error, the greater the importance of this input in the output. In this study, the input perturbation algorithm is implemented on the NNE model in order to perform the parameter sensitivity analysis of engineering structure. 


\section{Sensitivity analysis for parameters of prestressed concrete bridge}

\subsection{Application}

The proposed sensitivity method was applied for a prestressed concrete bridge, the detailed information about the bridge is shown in the paper by Lehký et al. (2017). The proposed method was utilized for determination of dominant input parameters of the prestressed concrete bridge. Such an information was used for proper set up of stochastic model and response surfaces for particular limit states and subsequent determination of selected uncertain design parameters followed by load-bearing capacity and reliability assessment. In this study, two limit states of the bridge were analyzed: (i) limit state of decompression (LSdec), and (ii) limit state of crack initiation (LScrack). A stochastic model with a total number of 21 random input variables corresponding to the material properties and loads was used. Each variable was defined using an appropriate probability distribution function (PDF), mean value and coefficient of variation (CoV). The detail description of parameters is shown in Lehký et al. (2017). In the case of NNE-based sensitivity analysis, the neural network with 21 neurons in the input layer and one neuron in the output layer, corresponding to the load-bearing capacity for each limit state (LSdec or LScrack) was used. Considering the performance aspects such as accuracy and convergence, some preselected neural networks are selected to form the NNE model on which the input perturbation algorithm is implemented to perform parameter sensitivity analysis.

A thousand random realizations of input variables were generated using the Latin hypercube sampling simulation technique using FReET probabilistic software (Novák et al., 2014). Numerical FEM analyses were performed repeatedly using ATENA software (Červenka et al., 2007) in order to obtain the structural response, i.e. the structure's normal load-bearing capacity in tons. Since the computational time needed to obtain load-bearing capacities related to the analyzed limit states is relatively high (one FEM simulation lasts about 15 minutes), an ANN-based surrogate model (Lehký and Šomodíkova, 2017) was created using obtained samples in order to significantly reduce the computational effort.

\subsection{Results}

The sensitivity factors and NNE performance factors were normalized to the value of the most dominant parameter. For LSdec, three dominant parameters were identified; see Fig. 2. Prestressing force $P_{1}$ is the parameter with high-level importance. The second important parameter is prestressing force $P_{2}$ with middle-level importance. The third important parameter is the secondary dead load $g_{1}$ with low-level importance. Other parameters have sensitivity lower than 0.1 ( 0.09 normalized) and can be declared non-dominant (zero-level importance).

For LScrack, six dominant parameters were identified, see Fig. 3. The parameters with high-level importance are the tensile strength of joints $f_{\mathrm{t}}$ and the prestressing force $P_{1}$. The parameter with middle-level importance is the prestressing force $P_{2}$. The parameters with low-level importance are the compressive strength of joints $f_{\mathrm{c}}$, the secondary dead load $g_{1}$ and the tensile strength of segments $f_{\mathrm{t}}$. Other parameters have sensitivities lower than 0.1 ( 0.1 normalized) and can be declared non-dominant.

\section{LSdec}

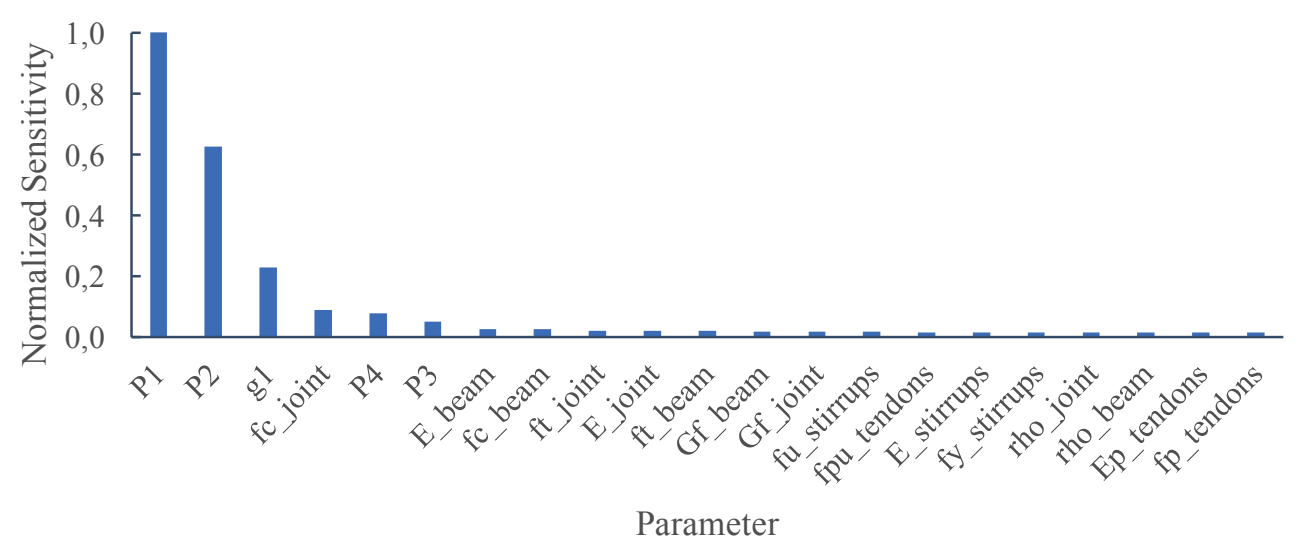

Fig. 2: Normalized sensitivity obtained by NNE-parameter sensitivity analysis for LSdec 


\section{LScrack}

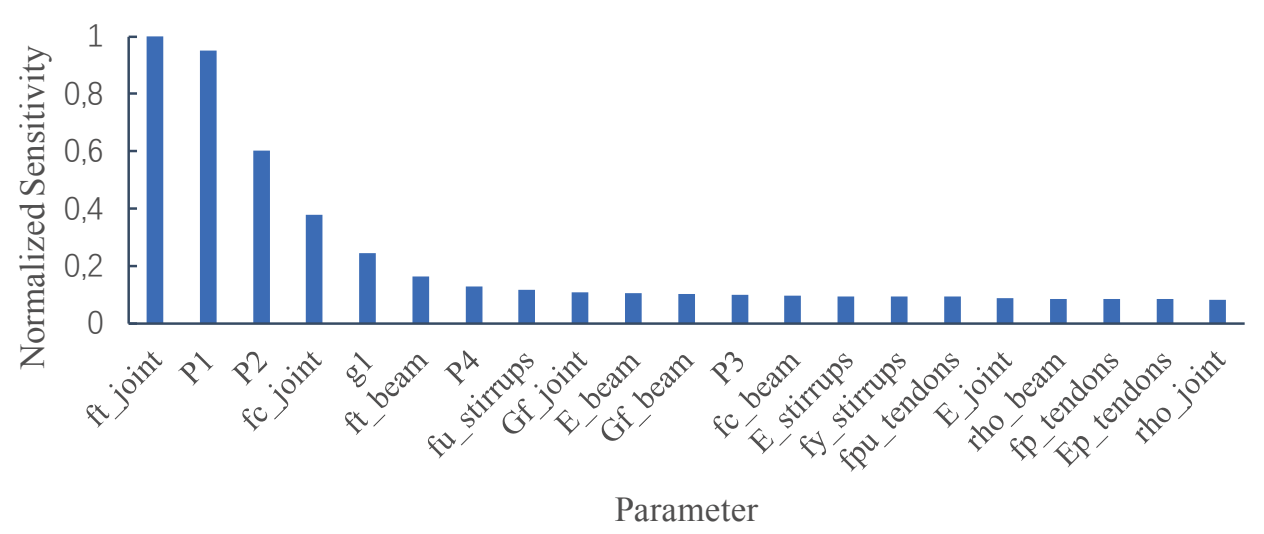

Fig. 3: Normalized sensitivity obtained by NNE-parameter sensitivity analysis for LScrack

\section{Conclusion}

NNE-parameter sensitivity analysis is an efficient and robust tool for detection of the dominant parameters of analyzed problem. When dealing with computationally demanding task such as prestressed concrete bridge analyzed in this paper, the utilization of surrogate models to approximate the original response function is strongly recommended in order to decrease computational effort.

\section{Acknowledgements}

The authors give thanks for the support provided by the Czech Science Foundation (GAČR) Project RESUS No. 18-13212S. First author thanks the Postgraduate Research \& Practice Innovation Program of Jiangsu Province Project No. KYCX17_0492 and the China Scholarship Council.

\section{References}

Benaouda, D., Wadge, G., Whitmarsh, R.B., Rothwell, R.G., MacLeod, C. (1999) Inferring the lithology of borehole rocks by applying neural network classifiers to downhole logs: an example from the Ocean Drilling Program. Geophysical Journal International, 136, 2, pp. 477-491.

Borgonovo, E. and Plischke, E. (2016) Sensitivity analysis: A review of recent advances. European Journal of Operational Research, 248, 3, pp. 869-887.

Červenka, V., Jendele, L. and Červenka, J. (2007) ATENA Program Documentation - Part 1: Theory, Cervenka Consulting, Prague, Czech Republic.

Cao, M.S., Pan, L.X., Gao, Y.F., Novák, D., Ding, Z.C., Lehký, D., Li, X.L. (2017) Neural network ensemble-based parameter sensitivity analysis in civil engineering systems. Neural Computing and Applications, 28, 7, pp. 1583-1590.

Kleijnen, J.P.C. (2010) Sensitivity analysis of simulation models: an overview. Procedia - Social and Behavioral Sciences, 2, 6, 7585-7586, doi: 10.1016/j.sbspro. 2010.05.130.

Lehký, D., Novák, D., Šomodíková, M., Pan, L.X. and Cao, M.S. (2017) A Comparison of Sensitivity Analyses of Prestressed Composite Bridge. 12th International Conference on Structural Safety \& Reliability (ICOSSAR 2017), Vienna, Austria, pp. 583-592.

Lehký, D. and Šomodíková, M. (2017) Reliability calculation of time-consuming problems using a small-sample artificial neural network-based response surface method. Neural Computing and Applications, 28, 1249-1263.

Maier, H.R. and Dandy, G.C. (2000) Neural networks for the prediction and forecasting of water resources variables: a review of modelling issues and applications. Environmental modelling \& software, 15, 1, pp. 101-124.

Novák, D., Vořechovský, M. and Teplý, B. (2014) FReET: Software for the statistical and reliability analysis of engineering problems and FReET-D: Degradation module. Advances in Engineering Software, 72, pp. 179-192. 